Venuta M. et al. (2021) Implementation of IoT Platform's Dashboards for the Visualisation of Dynamic KPIs: Insights from a Case Study. In: Camarinha-Matos L.M., Boucher X., Afsarmanesh H. (eds) Smart and Sustainable Collaborative Networks 4.0. PRO-VE 2021. IFIP Advances in Information and Communication Technology, vol 629. Springer, Cham. https://doi.org/10.1007/978-3-030-85969-5_48

\title{
Implementation of IoT Platform's Dashboards for the Visualisation of Dynamic KPIs: Insights from a Case Study
}

\author{
Marco Venuta ${ }^{1}$, Michela Zambetti ${ }^{1}$, Fabiana Pirola ${ }^{1}$, Giuditta \\ Pezzotta $^{1}$, Piergiorgio Grasseni ${ }^{2}$, Marco Ferrari ${ }^{2}$, Stefano Slavi ${ }^{2}$ \\ 1 Department of Management, Information and Production Engineering, \\ University of Bergamo, Viale Marconi, 24040, Dalmine, Italy \\ \{marco.venuta, michela.zambetti, fabiana.pirola, giuditta.pezzotta\}@unibg.it \\ 2 Smigroup, via C. Ceresa, 24015, San Giovanni Bianco, Italy \\ \{piergiorgio.grasseni, marco.ferrari, stefano.salvi\}@smigroup.net
}

\begin{abstract}
Nowadays, Internet of Things (IoT) platforms are becoming a huge opportunity for companies to collect data from connected machinery and analyse them to increase efficiency in production, optimize maintenance and introduce personalized service offerings. Specifically, multiple users can monitor real-time data and act based on updated information. Nevertheless, few studies are systematically focused on the implementation of Key Performance Indicators (KPIs) in the IoT environment. Based on an empirical case study, the article presents the implementation of dynamic KPI dashboards for an IoT platform, showing the challenges to face related to the trade-off between user desire and companies' technological readiness.
\end{abstract}

Keywords: KPI, IoT platform, implementation, case study

\section{Introduction}

Among the enabling technologies that are at the basis of Industry 4.0 and are transforming the traditional factories into smart factories, a prominent role is played by the Internet of Things (IoT) and its application in industrial contexts (IIoT) [1], [2]. An increasing number of companies are equipping the physical assets within their industrial environment with sensors, connecting devices to the Internet through IoT platform that manage the communication flow between machinery and users and provide applicationlevel capabilities for users to interact with the IoT system [3]. The adoption of IoT platforms enables the collection of a huge amount of data, that allows dynamic visualization of information and, among other metrics, of Key Performance Indicators (KPIs) [4]. Moreover, the implementation of advanced data analytics techniques, such as machine learning (ML) and artificial intelligence (AI) enables to identify future trends and detect potential problems [5]. The adoption of such a technology, therefore, deeply changes the role of KPIs and leads to an improvement in the overall performance and services of industrial companies by increasing the production efficiency, optimizing the maintenance interventions, and introducing personalized offerings for customers [6], [7]. In the literature, many studies in the field have focused their research on the development of ML and AI algorithms, while few describe how to implement an IoT platform and how to manage traditional KPIs in this new situation. Based on an 
$500 \mathrm{M}$. Venuta et al.

empirical case, the paper discusses the path towards defining an IoT platform, focusing on the development of the dynamic KPI monitoring and visualization, showing its usefulness in supporting companies in their decision-making process. KPIs are defined as dynamic due to their dynamic calculation and visualisation, through real-time data acquisition, and their flexibility in calculating targets and thresholds through automatically updated inputs. The article discusses how the implementation phase was undertaken and how it was impacted by the trade-off between specific desired functionalities of the user and actual technological possibilities of the firm. The paper is structured as follows: Section 2 presents the theoretical background that motivates the research. Section 3 presents the case study, where the implementation path for the development of dashboards for the visualization of dynamic KPIs. In Section 4 challenges and benefits regarding the dynamic KPIs implementation are presented. Finally, Section 5 provides conclusions and future development of the study.

\section{Background}

In recent years, companies are continuously looking for production systems characterised by excellent performance in terms of reliability, flexibility, sustainability and productivity [8], and the technologies within the Industry 4.0 paradigm represent an incredible opportunity to achieve these goals [9]. IoT, in particular, enables objects equipped with radio frequency identification, sensors, actuators to interact with each other and cooperate to achieve common goals [1]. Typically, to achieve a business opportunity like this, small and medium-sized enterprises, having limited skills and resources, need to join efforts in collaborative networks (CNs) [10]. A CN structure can provide an appropriate working environment for IoT because in this way different entities can share information in a facilitated way thanks to mutual trust. In this way, IoT assumes a key role in the provision of efficient and effective monitoring combined with an improvement in decision-making related to asset management [11]. Business monitoring is typically supported by an information system that provides information about several KPIs [12], which are powerful tools that relate enterprise data to business goals, enabling managers to guide the analytic process and identify deviations in their strategic plan [13]. The KPIs monitoring process always represented a challenging issue because of different factors:(1) large volumes and high speed of data that needed to be processed and analysed, (2) static calculation based on historical data, (3) not enough flexible visualization to quickly and accurately identify potential problems [6]. The adoption of technological advancements conveyed in IoT platforms allows users to monitor data in real-time (or near real-time) and make decisions based on KPIs calculated and visualized dynamically [14]. IoT platforms also enable to filter massive amounts of data and to parse out information and KPIs according to the different interests of the final users [6], [15]. Moreover, KPIs can be used to investigate the root causes of a problem and eliciting the future behaviour of the production thanks to the adoption of predictive algorithms [16]. An effective way of monitoring and evaluating performance is the selection of appropriate KPIs that strongly depends on the company's strategic intentions and its competitive environment [17], [18]. Several regulations as [19] [20] list the most common KPIs used by companies to monitor production and maintenance areas. The Overall Equipment Effectiveness (OEE) is the main KPI adopted in all the 
Implementation of an IoT Platform's Dashboards for the Visualisation of Dynamic KPIs 501

companies to monitor production performance. It identifies production losses [19] and their impacts [21] resulting in better use of available resources and therefore in optimization of production management and a reduction of associated costs. Besides OEE, other KPIs can be used to monitor production such as: Throughput rate, Production pace, Setup rate, etc. As far as maintenance monitoring is concerned, the most important and widely adopted KPIs are Mean Time Between Failures (MTBF), Mean Time To Failures (MTTF), Mean Time To Repair (MTTR). This family of indicators provide a synthetic measure of the expected time between two successive failures of an item, distinguishing between the expected time spent for repairing it and its expected functioning time [19]. Although automated process monitoring tools are accessible and already established performance measurements are available, there is a lack of proper processes to guide the transformation of raw data into applied knowledge [16], [22] and a lack of management tools to guide the process of capturing KPIs [23].

\section{Case Study}

In this paper, a case study methodology has been applied because, as indicated by [24], the intent is to illustrate new and innovative practices that organizations are adopting. The case study has been conducted in an Italian manufacturing company: SMI, one of the world's largest producers of bottling plants and packaging machinery. The company is characterized by a proactive attitude in the innovation field, and in recent years started an IoT project, initially focusing only on one of its machinery: the stretch-blow moulder, which transforms PET preforms into blown bottles. Through this project, SMI aims to collect operating data from all the machinery installed at the customer site and provide continuous real-time monitoring of industrial plants. This will allow them to enhance the decision-making process, improve asset and maintenance management and strategies. Moreover, by exploiting knowledge of the use phase of the machines, other objectives are related to the improvement of product design, customer profiling and service enlargement. All the information has been collected through participatory observation, which involved one of the researchers actively working on the achievement of the IoT solution. To develop this project, SMI has created a goal-oriented CN, characterised by a cooperative environment between different entities - SMI, university, technology providers and reseller, customers - working together to realise the IoT platform project. Thanks to this environment, many companies managers and external experts have been involved through semi-structured interviews and cross-department workshops to consider different points of view, and several established tools as Business Process Model and Notation (BPMN 2.0) and Failure Mode, Effects, and Criticality Analysis (FMECA) have been used to organize all the information collected [25], [26]. During the project collaboration, the researchers have elaborated an implementation path, that can be taken as the model for future IoT implementation works. The path can be divided into four main steps: (1) definition of the platform desired functionalities; (2) reengineering of the business processes affected by the platform; (3) development of the proof of concept of the platform dashboards (which represents the core of this paper); (4) implementation of the IoT platform with the connection of customer machines. At the beginning of the project, the different departments of the company have been asked to work on the definition of their needs and the desired functionalities that the platform 
$502 \mathrm{M}$. Venuta et al.

should include (phase 1). This work has also been supported with an analysis of the company business processes (phase 2), to understand the peculiarities of SMI's processes and identify the areas and departments that could mostly be impacted and benefit from the introduction of IoT. Among the desired functionalities, the visualization of metrics related to production and maintenance performance emerged as fundamental to monitor the machinery functioning, but also to define internal business performances and using them to define targets and continuous improvement strategies. The proof of concept of platform dashboards (phase 3) contains all the information, data and KPIs that the endusers want to manage in the platform. To achieve this outcome, several activities have been carried out in this third phase. First, an FMECA on the machine has been carried out to understand the main critical components. Then, based on these results and the functionalities previously defined, the machinery data to monitor has been selected. Particularly, the company has recognised that not only machine-specific telemetry data (e.g. temperature, vibration, peak current, electric consumption) plays a central role in the machine monitoring process, but also production and maintenance KPIs. Indeed, the role and the calculation of traditional KPIs change with the introduction of IoT, shifting from a static and always historical analysis towards dynamic calculation thanks to realtime data and data analytics algorithms. To define which KPIs include in the proof of concept, a literature analysis on most adopted KPIs has been conducted. To this purpose, the most common standards [19], [20] have been taken into account. The KPIs report has been presented to the internal users, who then evaluated each KPI considering the theoretical benefits and all the possible uses in the SMI context.

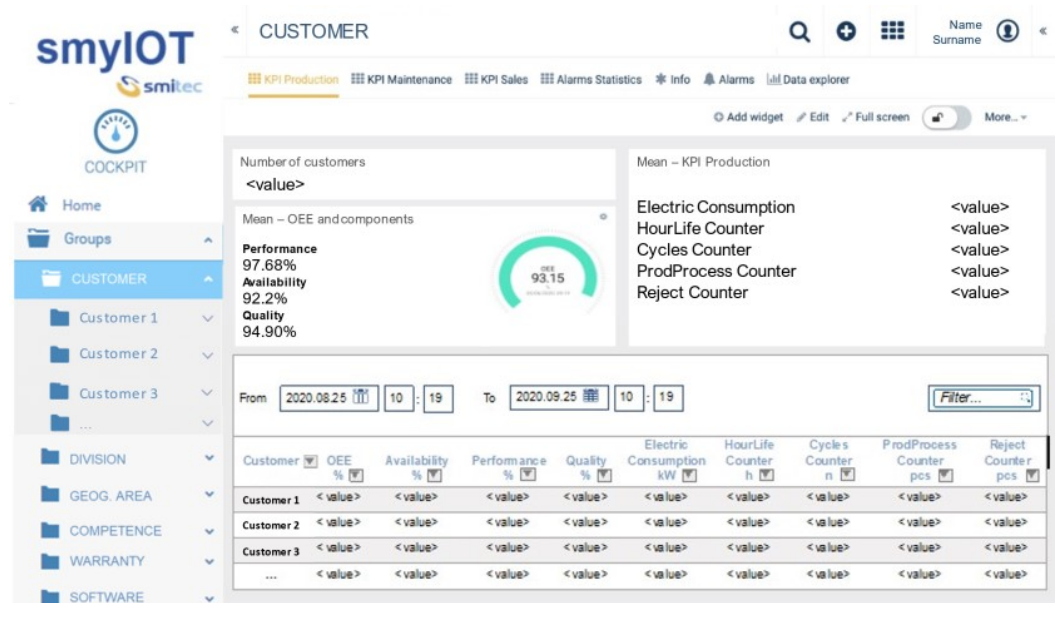

Figure 1. SmyIoT dashboard example with fictitious values

Before starting the development of the proof-of-concept, a study on how the platform is structured has been performed. The platform is characterized by a recurrent hierarchical structure. Starting from the homepage dashboard, the user can explore the platform in different ways, using the direct link on the dashboard or using the navigation menu with the button "GROUPS". Groups are organized in a hierarchical way and, gradually deepening the analysis into connected subgroups, it is possible to obtain information and statistics related to smaller samples of devices, until the visualization of the dashboards of a single machine. Each machine can be reached by the exploration of all groups since 
Implementation of an IoT Platform's Dashboards for the Visualisation of Dynamic KPIs 503

it is assigned to more than one group using a one-to-many logic. This structure combined with the scalability property of the KPIs enables the creation of a realistic proof of concept; it represents several standard dashboards that contain all the information requested by the users and can be applied in all the different hierarchical level of the platform. Based on these characteristics, preliminary proof of concept has been developed. Figure 1 shows an example of a dashboard; it depicts the KPIs and data selected in order to monitor, in the specific case, the aggregate production of the customers. The same dashboard can be used at each level of the hierarchical structure: the framework and KPIs will be replicated but their calculated values, targets and thresholds will change depending on the level of aggregation and trends of input data. Before the implementation phase, some important considerations of the prioritisation and feasibility must be done. All the data and the KPIs selected until this point represented the desires of the internal-end users, without considering technological and economical constraints. Thus, through several interviews, the software developers validated the feasibility of the proof-of-concept requests, considering what the company technological readiness permitted and what was feasible. Given the central role of the KPIs, also their implementation feasibility evaluation was crucial. So, four steps have been identified:

1. Theoretical analysis: define how the KPIs are calculated theoretically

2. Analysis of data availability: define what the company already has, and which data is missing

3. Analysis of possible solutions: define how to collect the necessary missing data and its feasibility

4. Definition of the implementation priority: select what should be implemented and in which order, also considering the component FMECA criticality

An explanatory case of the implementation feasibility evaluation is reported in Table 1, considering the OEE.

Table 1. OEE Analysis

Steps Description

OEE is a synthetic indicator of the effectiveness of the work units. Its formula is $\mathrm{OEE}=\mathrm{AxPxQ}$ which represents the actual production ratio net of the time losses:

$\boldsymbol{A}=$ Availability It considers the plat uptime considering time losses for setup,

1 breakdowns, and corrective maintenance.

$\boldsymbol{P}=$ Performance It considers the plat uptime considering speed losses and detects time losses due to minor stops and waiting times.

$\boldsymbol{Q}=\boldsymbol{Q}$ uality It considers the plat uptime considering losses due to defective parts production and rework.

SMI, being already oriented to the Industry 4.0 , has implemented in its machines its OEE formula to answer to the characteristics of the specific reference market. The OEE is calculated as a global index for the whole machine, without distinguishing the recipe used and with a simplification regarding the counting of relative times (planned shutdown time, setup time, etc.). Furthermore, at the machinery level, events and times related to shutdowns for maintenance and set-up operations are not detected and classified because such detailed calculations and analysis are typically left to higherlevel factory IT applications. 
$504 \mathrm{M}$. Venuta et al.

\begin{abstract}
This state of the art has indicated the need for specifics enrichments of the current machinery dataset, not only considering the machine level but also all IoT stack. It will be possible to collect and classify data according to the used recipe, link single machine 3 state and alarms to specific machine components and track related occurrences and timing. Furthermore, to collect set-up and maintenance time it will be necessary to define and implement an appropriate IT infrastructure to interact with operators and technicians to collect this information from their feedbacks.

SMI decided to improve the current OEE by adding the recipe and components identification, due to its low software development effort and its importance for the internal users. SMI decided to not take into consideration the set-up time, although operators could provide this information with active feedback, currently, they are not engaged in these kinds of activities. Therefore, SMI needs to provide an unfailing system to obtain them, but its costs are high and there is no implementation urgency.

After this analysis, SMI decided to keep the previously defined OEE formula, but improving it, adding the possibility to exploit the scalability potential through the detection of recipes used and components effectiveness. Some of the issues raised for the OEE analysis were also visible in the calculation of others KPIs. In particular, given the actual difficulty of collecting and interpreting in the right way data about maintenance intervention traceability, SMI decided that, at the current state, the MTBF would be considered later. In fact, even if MTBF is a useful KPI, the effort needed to detect maintenance interventions would have slowed down the IoT project excessively. At this point, the software developers had all the information to develop the IoT platform (phase 4). The implementation phase followed an agile approach characterized by flexibility and adaptability principles, to maintain the platform open to modification and extensions, always supported by technology provider personnel. SMI started to collect data to feed the platform from the machines of the showroom and test department allowing the developers to get responsive feedback on their work, and improve the platform features one piece at a time until the ultimate platform version has been installed in a set of SMI machinery.
\end{abstract}

\title{
4 Discussion
}

The development of the IoT platform highlights several challenges that SMI had to face for the successful implementation of dynamic KPI, that can be generalized for other companies too. Overall, it is possible to notice that there are potential issues related to the complete availability of data, since the machine protocol often does not include the necessary data to compute specific metrics. The collection of these data may require software developments, the selection of proxy or the development of a system that enables operators to collect feedbacks. Considering this last choice, the level of confidence in the machine operators is still low, since they are not usually prone to use such solutions, not considering them essential for their daily work; so, it is clear that there is a need to improve a digital culture and a proactive mentality at all levels of $\mathrm{CN}$ companies. In particular, this culture must emerge among end-customers, because they are a key part of the data collection; if the customer does not understand the usefulness and importance of this data, it may not be inclined to collaborate effectively. However, once the right data and KPIs are implemented in the platform, they will be accessible in real-time, will increase machine reliability, and will be scalable at different levels, 
Implementation of an IoT Platform's Dashboards for the Visualisation of Dynamic KPIs 505

paying back the efforts required for their collection and calculation. The real-time computation will enhance the monitoring of production and the recognition of problems, as well as the definition of the responsibility when a certain KPI lowers under predefined limits. In addition, it will be easy to carry out benchmarking analyses on machine performance, which can be used, among other options, to improve machine design. The chance to access real customer data will enhance the possibility to show the performance of machinery to increment sales, but also to introduce new business models and customized solutions thanks to better customer profiling. Another interesting utilization is the opportunity to compare machinery performance over time and give evidence of the enhancement achieved after maintenance interventions or adopting preventive maintenance approaches.

\section{Conclusion and Future Research Direction}

Thanks to the CN collaboration, SMI was able to quickly develop an IoT platform for the dynamic visualisation of KPIs; having a common goal, the entities cooperated effectively by leveraging the network's expertise to overcome challenges. In future, the $\mathrm{CN}$ will be able to exploit the IoT platform to strengthen communication between its parts. However, the work does not come without limitations: even if the proposed work has been proven to be effective for the reference company, multiple applications are recommended to reach a higher level of generalisation. Because of the initial state of activities, the most severe limitation of the project is represented by the absence of numerical data that represent the platform's benefits. In parallel with the continuous development of the platform functionalities future research directions have been defined. First of all, there is the need to define ways to create a digital culture and mentality for the use of platforms, or to improve it where it is already present. Besides the qualitative evaluation of benefits, which may be more attractive for managerial departments rather than the operative users, and traditional training sessions to explain tools and their benefits, one suggestion could be to work on the gamification of specific platform parts, to involve the end-users with real incentives. Moreover, a new way to conduct the development process should be conceptualised and introduced in the industrial setting including both user-centric and technology-driven perspective and competencies, considering the need to understand the technological dimension related to digital components of the machinery, and the user-centric dimension.

\section{Acknowledgement}

The researchers of the University of Bergamo have been funded by the Erasmus + Knowledge Alliance project DIGIFoF (Digital Design Skills for Factories of the Future - Project Nr. 601089-EPP-1-2018-1-RO-EPPKA2-KA)

\section{References}

1. J. Dalzochio et al., «Machine learning and reasoning for predictive maintenance in Industry 4.0: Current status and challenges», Computers in Industry, vol. 123, pag. 103298, dic. 2020

2. S. Schneider, «THE INDUSTRIAL INTERNET OF THINGS (IIoT): APPLICATIONS AND TAXONOMY», pag. 42, 2017.

3. I. Machorro-Cano, G. Alor-Hernández, N. Cruz-Ramos, C. Sanchez-Ramirez, e M. SeguraOzuna, «A Brief Review of IoT Platforms and Applications in Industry», 2018, 293-324. 
$506 \mathrm{M}$. Venuta et al.

4. P. Moens et al., «Scalable Fleet Monitoring and Visualization for Smart Machine Maintenance and Industrial IoT Applications», Sensors, vol. 20, n. 15, pag. 4308, ago. 2020.

5. M. Subramaniyan, A. Skoogh, H. Salomonsson, P. Bangalore, e J. Bokrantz, «A data-driven algorithm to predict throughput bottlenecks in a production system based on active periods of the machines», Computers \& Industrial Engineering, vol. 125, pagg. 533-544, nov. 2018.

6. A. Mate, K. Zoumpatianos, J. Mylopoulos, T. Palpanas, E. Koci, e J. Trujillo, «A Systematic Approach for Dynamic Targeted Monitoring of KPIs», pag. 15, 2014.

7. T. Pauli e Y. Lin, «The Generativity of Industrial IoT Platforms: Beyond Predictive Maintenance?», pag. 7, 2019.

8. G. Ante, F. Facchini, G. Mossa, e S. Digiesi, «Developing a key performance indicators tree for lean and smart production systems», IFAC-PapersOnLine, vol. 51, 13-18, gen. 2018

9. Y. Lu, «Industry 4.0: A survey on technologies, applications and open research issues», Journal of Industrial Information Integration, vol. 6, pagg. 1-10, giu. 2017.

10. L. M. Camarinha-Matos, H. Afsarmanesh, N. Galeano, e A. Molina, «Collaborative networked organizations - Concepts and practice in manufacturing enterprises», Computers \& Industrial Engineering, vol. 57, n. 1, pagg. 46-60, ago. 2009.

11. M. Syafrudin, G. Alfian, N. L. Fitriyani, e J. Rhee, «Performance Analysis of IoT-Based Sensor, Big Data Processing, and Machine Learning Model for Real-Time Monitoring System in Automotive Manufacturing», Sensors (Basel), vol. 18, n. 9, set. 2018.

12. D. Parmenter, «Key Performance Indicators: Developing, Implementing, and Using Winning KPIs, 4th Edition |Wiley», Wiley.com, 2010.

13. M. Badawy, A. A. A. El-Aziz, A. M. Idress, H. Hefny, e S. Hossam, «A survey on exploring key performance indicators», Future Computing and Informatics Journal,vol.1,47-52, 2016.

14. O. Mörth, M. Eder, L. Holzegger, e C. Ramsauer, «IoT-based monitoring of environmenta conditions to improve the production performance», Procedia Manufacturing, vol 45, 2020.

15. M. Mahmoodpour, A. Lobov, M. Lanz, P. Makela, e N. Rundas, «Role-based visualization of industrial IoT-based systems», in 2018 14th IEEE/ASME International Conference on Mechatronic and Embedded Systems and Applications (MESA), Oulu, lug. 2018, pagg. 1-8.

16. A. Papacharalampopoulos, C. Giannoulis, P. Stavropoulos, e D. Mourtzis, «A Digital Twin for Automated Root-Cause Search of Production Alarms Based on KPIs Aggregated from IoT», Applied Sciences, vol. 10, n. 7, pag. 2377, mar. 2020.

17. V. Jovan e S. Zorzut, «Use of Key Performance Indicators in Production Management», in 2006 IEEE Conference on Cybernetics and Intelligent Systems, giu. 2006, pagg. 1-6.

18. G. Hwang, J. Lee, J. Park, e T.-W. Chang, «Developing performance measurement system for Internet of Things and smart factory environment», International Journal of Production Research, vol. 55, n. 9, pagg. 2590-2602, mag. 2017.

19. «ISO22400 KPI Manufacturing operations management, Key performance indicator». 2011.

20. «prEN 15341 Maintenance - Maintenane Key Performance Indicators». BSi, 2006.

21. S. Nakajima, Introduction to TPM: Total Productive Maintenance. Productivity Press, 1988.

22. H. Miller e P. Mork, «From Data to Decisions: A Value Chain for Big Data», IT Professional, vol. 15, pagg. 57-59, gen. 2013.

23. S. Softic, E. Lüftenegger, e I. Turcin, «Tracking and Analyzing Processes in Smart Production», 2020, pagg. 37-50.

24. R. W. Scapens, «Researching management accounting practice: The role of case study methods», The British Accounting Review, vol. 22, n. 3, pagg. 259-281, set. 1990.

25. C. Voss, N. Tsikriktsis, e M. Frohlich, «Case Research in Operations Management», International Journal of Operations \& Production Management, vol. 22, 195-219, 2002.

26. R. K. Yin, Case Study Research: Design and Methods. SAGE Publications, 1984 